\title{
INTEGRAL Earth occultation observations revealing the soft gamma ray background - The operational view
}

\author{
Jutta M. Hübner ${ }^{1}$ \\ ESA/ESOC \\ Darmstadt, Germany \\ email: jutta.huebner@esa.int
}

Carmen Lozano

VEGA Space GmbH

Darmstadt, Germany

email: carmen.1ozano@esa.int

\section{Richard Southworth \\ ESA/ESOC \\ Darmstadt, Germany \\ email: richard.southworth@esa.int}

\begin{abstract}
Cosmic rays interacting with the Earth's atmosphere can generate photons with energies up to the gamma regime. This effect has only been studied marginally from space. In 2006, four short Earth occultation observations were performed successfully with INTEGRAL during a solar minimum, where the gamma-ray flux of the Earth's atmosphere was at a maximum due to cosmic ray bombardment. These observations demonstrated the technical feasibility of the Earth occultation observation method with INTEGRAL and its high scientific potential.
\end{abstract}

Exploiting further this scientific potential, another set of occultation observations is planned for AO-9 (2012-2013) to further probe the three distinct soft gamma-ray diffuse components: the cosmic X-ray background (CXB), the galactic background emission and the Earth atmospheric emission due to both the reflection of the CXB and cosmic-ray interactions. The total exposure time of the AO-9 observations will be longer than in 2006. In order to follow the evolution of the soft gamma-ray diffuse components towards the solar maximum, the upcoming observations will be spread over a period of two years.

This paper summarises the scientific objectives of the planned Earth occultation observation campaign with INTEGRAL as well as the associated operational strategies and challenges, such as the need to use a non-standard INTEGRAL pointing mode.

The Extreme and Variable High Energy Sky,

Chia Laguna (Cagliari), Italy

September 19-23, 2011

\footnotetext{
$1 \quad$ Speaker
} 


\section{Introduction}

ESA's INTErnational Gamma Ray Astrophysics Laboratory (INTEGRAL), launched in 2002, is designed to perform multispectral observations of the most violent and exotic objects of the universe [1]. Nevertheless, in 2004 it was proposed to use INTEGRAL to probe cosmology by using the Earth as an occultation screen to further characterize the soft gamma-ray diffuse components. Consequently, a set of Earth occultation observations (EO) was performed with INTEGRAL in 2006. The main challenge during the planning of these observations was to overcome the attitude constraint of INTEGRAL while still using nominal operational software and standard safety checks. This paper summarises the EO campaign performed with INTEGRAL in 2006 as well as the studies for the second EO planned for 2012-2013.

\section{Scientific objective of EO: The diffuse high energy radiation}

The scientific justification of the EO with INTEGRAL in 2006 was to further characterize the three distinct soft gamma-ray background components: the cosmic X-ray background (CXB), the galactic background emission (i.e., the apparently diffuse component most probably due to faint compact objects in our galaxy) as well as the Earth's atmospheric emission, which consists of two components, the reflections of CXB on the Earth's atmosphere and cosmic-ray interactions.

The origin of the diffuse high-energy radiation with energies above $20 \mathrm{keV}$ is thought to be mainly unresolved active galactic nuclei (AGN) but also super-massive and accreting black holes extending to cosmological distances with a contribution from type Ia supernovae in the low-energy gamma range [2]. Even though space is filled with this diffuse high-energy emission, it has only been studied marginally: while up to $80 \%$ of the diffuse emission below 10 $\mathrm{keV}$ could be resolved into point sources [3,4], only $2.5 \%$ of the radiation with $\mathrm{E}>20 \mathrm{keV}$ is resolved [5]. Especially the spectral shape and normalization around the peak of the CXB emission between $20-60 \mathrm{keV}$ is not determined very accurately but is crucial to estimate the AGN fraction, which is thought to contribute significantly in this spectral range [6].

\section{Method to study the CXB with INTEGRAL}

The CXB can be studied via the masking technique, where the Earth is used as a screen that occults a part of the diffuse high energy emission. By measuring the drop of the background flux as the Earth moves across the field of view (FoV), the CXB flux can be measured in an indirect way. Due to its high sensitivity in the $17-500 \mathrm{keV}$ range and its large FoV, INTEGRAL is well suited to perform such observations. Therefore, in 2004 it was proposed to perform EO with INTEGRAL. 


\section{Challenges and operational implementation of EO}

During routine operations, the payload is not allowed to point to the Earth within $15^{\circ}$ ("attitude constraint") for two reasons: this way, the observations won't be affected by the Earth albedo. But even more importantly, this prevents the star trackers, which are co-aligned with the payloads' boresight, to be blinded by the Earth. Thus they can be used for attitude control.

Contrarily, during the EO the payload has to be aligned with the Earth at some point. Therefore, the attitude constraint is the most important operational constraint for the EO. While INTEGRAL is highly autonomous for routine operations, the main challenge for these nonroutine observations is to enable INTEGRAL to point to the Earth - and therefore violate the attitude constraint - while assuring the controlled and safe operation of spacecraft and payload. In order not to compromise the safety during the EO, a method for planning the operations had to be applied that would fulfill the nominal attitude constraints to allow the use of the nominal operational software and the standard safety checks during revolution planning. Additionally, the following requirements had to be met: The EO should be performed in the ascending part of the orbit, to maximise the time available before entering the radiation belts ${ }^{2}$ in case a recovery is necessary. It should take place as early as possible after exiting the radiation belts and reconfiguration of the instruments, to obtain a large apparent Earth size as occultation screen. Furthermore, no strong sources should be within the FoV, to reduce contamination by foreground sources.

Since INTEGRAL has no on-board telemetry storage and schedule, it is controlled and commanded from ground via a pre-planned automatic command schedule. This so called timeline contained the following spacecraft maneuvers for the EO in 2006: In the descending part of the orbit, a slew (slew \#1) was planned to the EO attitude (attitude \#1, unconstrained at this time), where the Earth would pass through the instruments' FoVs after perigee. Since this attitude would be constrained after perigee, another slew (slew \#2) was planned before perigee to change the attitude to an unconstrained one. In the ascending part of the orbit, when attitude \#1 was constraint free again, another slew (slew \#3) was planned back to attitude \#1.

Thus, planning wise, a nominal timeline was generated and the nominal operational software and standard safety checks were executed. However, no EO would take place with this timeline. All planned activities are illustrated in Figure 1 (red numbers).

For enabling the Earth to pass through the instrument's FoV, the following method was applied: after having reached attitude \#1 for the first time, the commanding to INTEGRAL from the timeline was inhibited, i.e., the next attitude change (slew \#2) was not executed. Therefore, INTEGRAL was still in attitude \#1 during perigee and the Earth crossed the instrument's FoV in the ascending part of the orbit. These activities did not pose any risk to INTEGRAL, since the spacecraft's autonomous control mechanisms are designed to cope with a situation like this, which is comparable to having lost the ground contact just before the execution of slew \#2. To

2 During the passage of the van Allen radiation belts around perigee, the four scientific instruments aboard INTEGRAL are in a safe configuration to avoid damages from the particles trapped in the radiation belts. 
keep a safe and controlled attitude while the star trackers are blinded, the prime star tracker was removed from the control loop and a different guidance mode was used: yaw control was transferred to the gyro and the pitch and roll control to the fine sun sensor. Furthermore, repeated star tracker mappings were executed to increase the attitude reconstruction accuracy a posteriori. Once the attitude was unconstrained again, the star tracker was re-configured for control and a manoeuvre was executed to correct possible yaw drift and to re-join nominal operations. The executed activities are also illustrated in Figure 1 (green numbers).

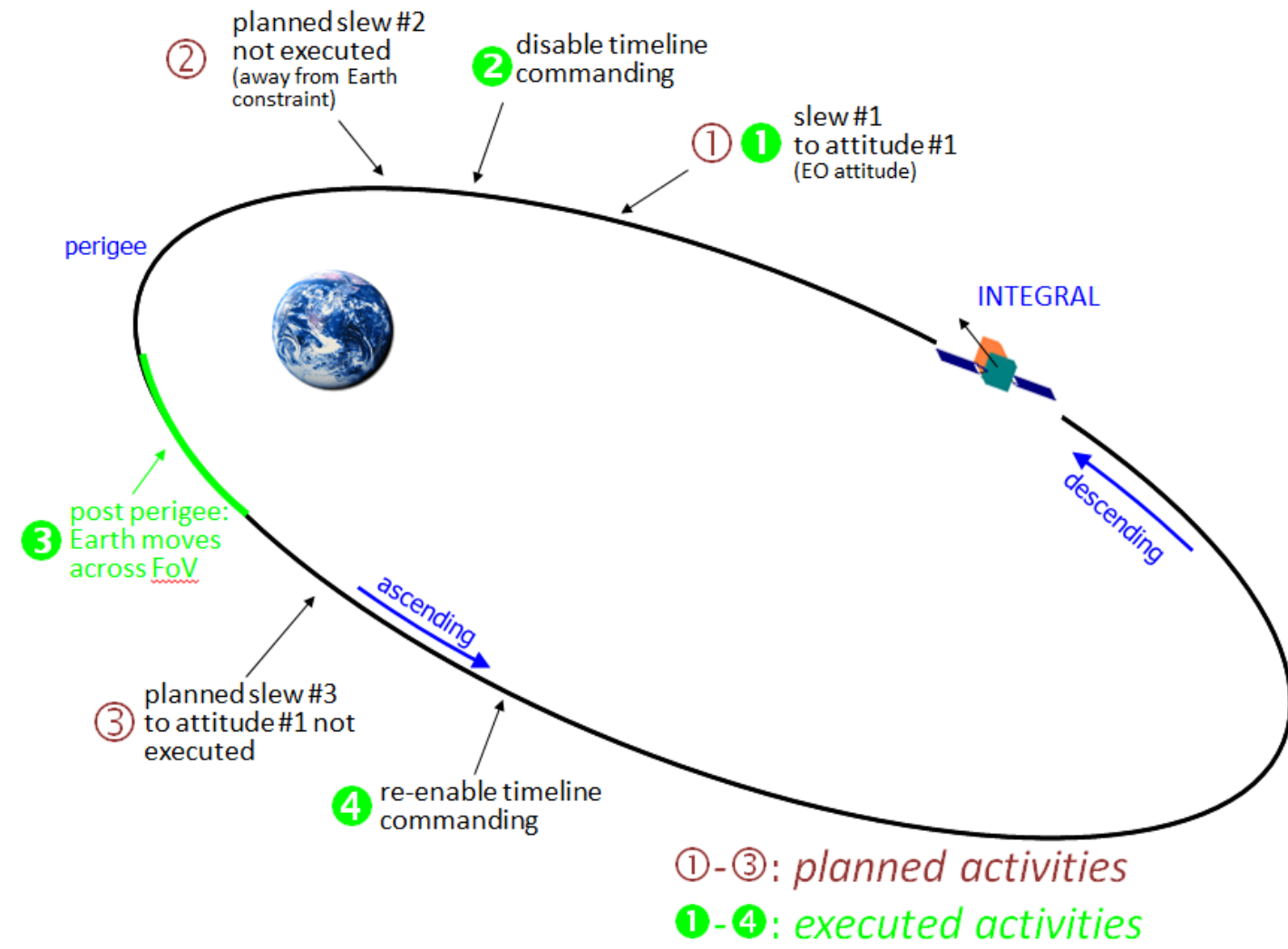

Figure 1: Operational planning (red numbers) and execution of activities (green numbers) during EO. For further details, please refer to the text.

\section{Results and limitations of the first EO}

The observations performed in 2006 demonstrated the technical feasibility of the Earth occultation observation method with INTEGRAL and its high scientific potential: A clear signal was observed and it was possible to derive the spectrum of the three fundamental components. Furthermore, the soft gamma-ray diffuse components could be separated from the instrumental effects and the instrumental background by precise modeling. The contribution from known point sources within the FoV could be subtracted as well. The scientific results were satisfactory, fulfilled the expectations and were consistent with historic results $[6,7,8]$. 
The EO in early 2006 had relatively short exposure times (the sequence described above was repeated four times during four orbits resulting in four exposures of $60 \mathrm{ks}$ in total within 16 days) limiting the statistical quality of the data. Furthermore, the EO took place during the solar minimum, where the disturbing high energy emission from the Earth due to cosmic ray interactions was at a maximum. Therefore, another series of EO with INTEGRAL is planned for 2012-2013 to confirm the previous results with improved statistics and to refine the existing models.

\section{Constraints for the second EO}

For the second set of EO, more ambitious and challenging requirements are defined:

- Period of observations: The EO should be performed around the solar maximum (predicted for early 2013) to reduce the high-energy emission of the Earth atmosphere above $70 \mathrm{keV}$. Additionally, it should be spread over a period of two years to follow the evolution of the soft gamma-ray diffuse components towards the solar maximum for evaluating the dependency of the Earth emission on the solar cycle.

- Desired target regions: Different areas inside the galactic plane region (GR for Galactic Ridge) and outside the galactic plane region (NGR for Non Galactic Ridge) should be observed. Bright sources shall be avoided to reduce the contamination by foreground sources.

- Longer exposure times and more observations shall be executed.

- On-Off-On-pointing requirement: The time with the Earth inside the FoV shall be $\sim 50 \%$ of the total EO duration.

\section{Conclusions for the second EO}

Studies on the target visibility and the observation duration were performed in the context of the upcoming EO starting in May 2012. The galactic regions against which the Earth is visible vary enormously over the years due to the orbital evolution (see Figure 2). While the GR 1 and 2 are only observable in the descending part of the orbit shortly before perigee, the NGR targets are highly constrained until mid-2013. 


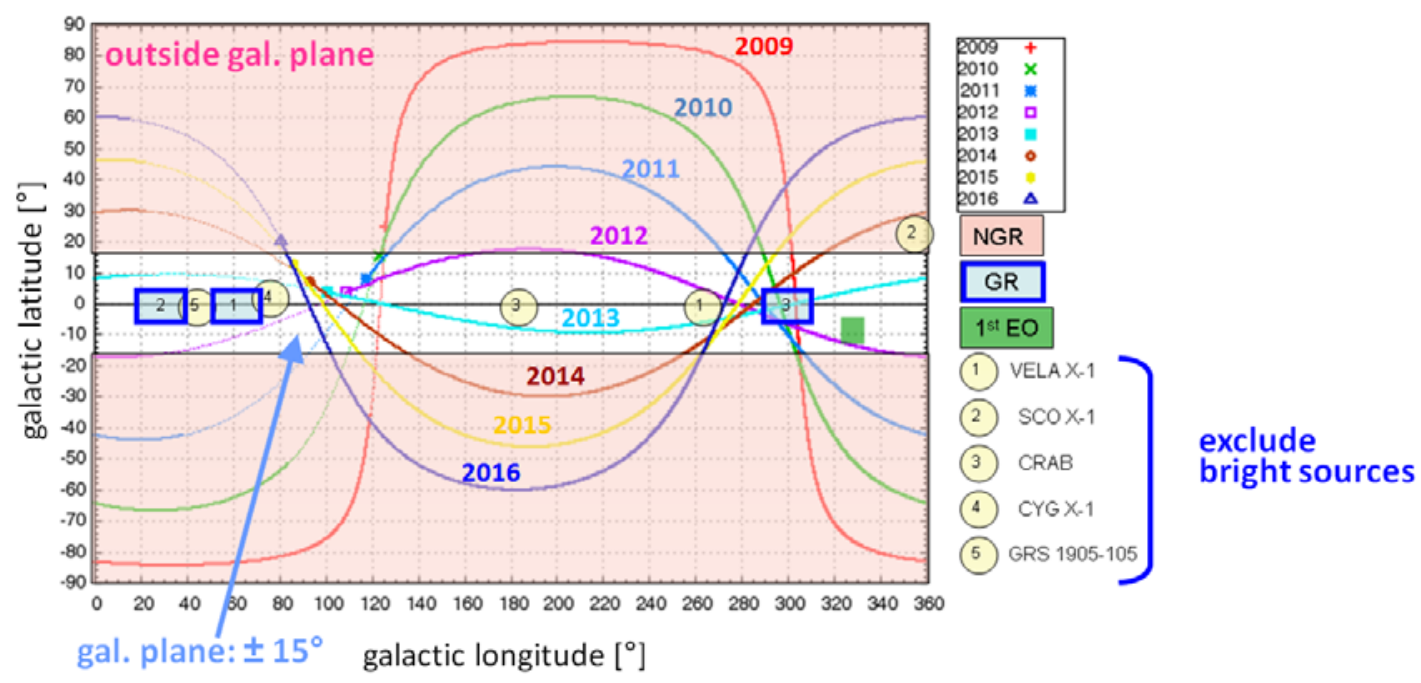

Figure 2: Position of the center of the Earth as seen from INTEGRAL in the galactic coordinate system as a function of time (the first orbit of each year is illustrated by a colored line; the icons indicate the positions of the respective perigee): Observations inside (GR, marked in white) and outside the galactic plane (NGR, marked in red) have been requested. For GR, particularly the blue areas are of interest. The influence of bright sources (yellow circles) shall be avoided.

The duration of the Earth passage through the different FoVs is shown in Figure 3. For a duration of the Earth passage of 6 hours within the IBIS FoV, the minimum observation duration is $\geq 12.5 \mathrm{~h}$ due to the star tracker constraint (black line). At apogee, the Earth is within the SPI FoV for a duration of about $50 \mathrm{~h}$. Therefore, as a result of the on-off-on pointing requirement of $50 \%$, the minimum Earth observation would be about $100 \mathrm{~h}$, which is much longer than a revolution.

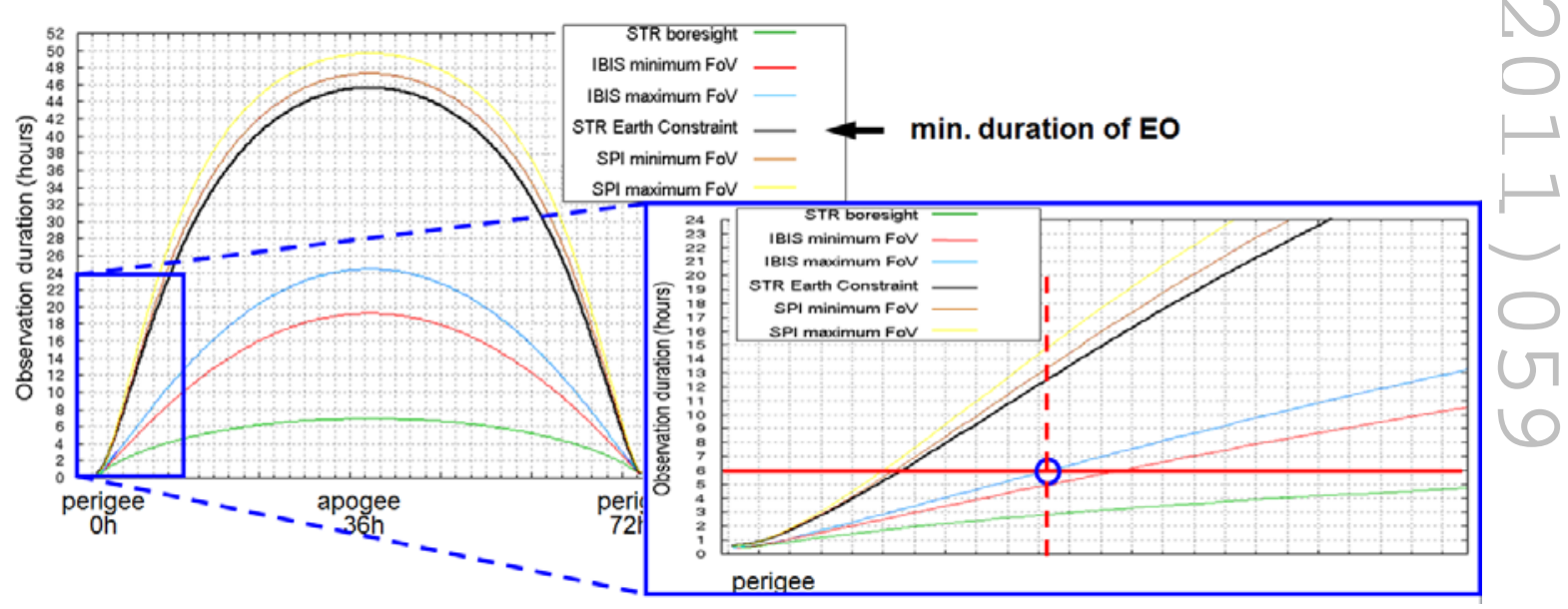

Figure 3: Duration of the Earth passage through the different FoVs around the center of the observation. The black line illustrates the star tracker Earth constraint, i.e., the minimum observation duration. On the right, there is a zoomed in view of the rising part of the orbit. 
16 observations of about $30 \mathrm{ks}$ each will be performed during the second EO. They will be spread over several years around the solar maximum. Just like during the first EO, the Earth will pass through the instrument's field of view post perigee. The expected result from the second series of Earth occultation observations is to determine the CXB with improved statistics and less systematics. Furthermore, the dependency of the Earth's emission on the solar cycle can be studied.

\section{Acknowledgments}

The authors would like to thank Peter Kretschmar (ESA/ESAC), Roman A. Krivonos (MPA/IKI), Alfonso Sancho (GMV) and Marc Türler (ISDC Data Center for Astrophysics) for the fruitful discussions. INTEGRAL is an ESA project. We are grateful to ASI, CEA, CNES, DLR, ESA, INTA, NASA and OSTC for support.

\section{References}

[1] Winkler, C., et al., 2003, A\&A 411, L1-L6

[2] Zdziarski, A. A., 1996, MNRAS, 281, L9

[3] Brandt, W. N. \& Hasinger, G., 2005, ARA\&A, 43, 827

[4] Gilli, R., Comastri, A. \& Hasinger, G., 2007, A\&A, 463, 79

[5] Paltani, S., et al., 2008, A\&A 485707

[6] Churazov, E., et al., 2007, A\&A, 467, 529

[7] Churazov, E., et al., 2008, MNRAS, 385, 719

[8] Türler, M., et al., 2010, A\&A, 512, A49 